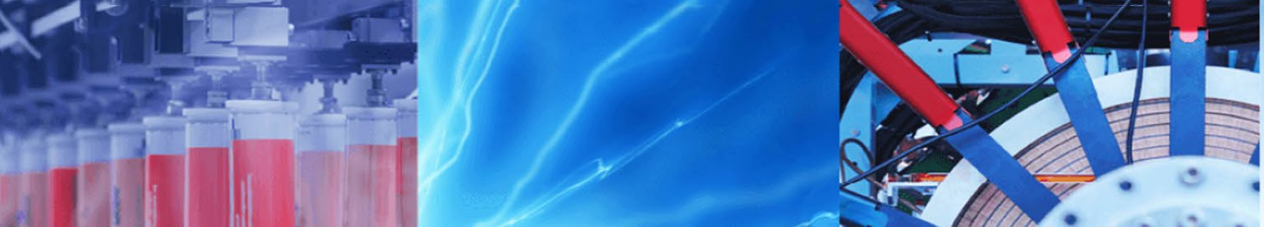

Research Article

\title{
Doubly fed induction generators to enhance inter-area damping based on a Robust controller: $\mathrm{H}_{2} / \mathrm{H}_{\infty}$ Control
}

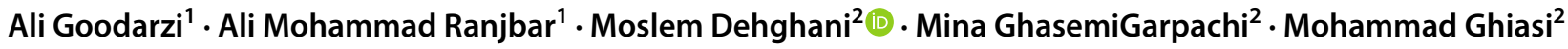

Received: 26 January 2020 / Accepted: 4 January 2021 / Published online: 11 January 2021

(C) The Author(s) 2021 OPEN

\begin{abstract}
In this study, an auxiliary damping controller based on a robust controller considering the active and reactive power control loops for a doubly-fed induction generator for wind farms is proposed. The presented controller is able to improve the inter-area oscillation damping. In addition, the proposed controller applies only one accessible local signal as the input; however, it can improve the inter-area oscillation damping and, consequently the system stability for the various working conditions and uncertainties. The oscillatory modes of the system are appointed using the linear analysis. Then, the controller's parameters are determined using the robust control approaches $\left(H_{\infty} / H_{2}\right)$ with the pole placement and linear matrix inequality method. The results of the modal analysis and time-domain simulations confirm that the controller develops the inter-area oscillation damping under the various working conditions and uncertainties.
\end{abstract}

Keywords Doubly-fed induction generator · Robust controller · Inter-area oscillations · Power system stability

\section{Introduction}

The increasing development of the wind energy-based applications imposes new challenges on the stability and performance of the power systems. Nowadays, among the various wind turbine topologies, the variable-speed wind turbines with the doubly-fed induction generators (DFIGs) are preferred due to its capability in maximum power tracking also grid side converter facilitates the power exchange through the rotor converter, in addition provides additional reactive power support [1]. Most of the electromechanical oscillations arise from the synchronous machines [2]. Physically, these oscillations result from the interaction between the turbine-to-rotor mechanical torque and the stator-to-rotor electrical torque. In the local modes, one generator may oscillate dissimilarly toward the other ones. This oscillation affects both the generator and the transmission line. So that, the neighboring groups of generators may oscillate in different manners. It is because of the fact that there is a low power-transmission capability between the groups of generators.

In fact, the shaft speed of the generators is different from the speed of the rotating field (caused by threephase mains voltages) and fluctuations. If the shaft speeds of a group of generators close to each other do not fluctuate relative to each other, and a group of other generators do the same, then the shaft speeds of the generators of these two groups fluctuate relative to each other, this phenomenon is called interregional fluctuations.

The high penetration levels of the wind power can affect the dynamic behavior of the system's electromechanical oscillations. Also, the control capability of the wind turbines can be applied to improve the stability of the power systems oscillations. In this case, besides improving the dynamic behavior of the wind turbine, increasing the capability of the power system oscillations damping is considered in the control process of the wind turbine.

Moslem Dehghani, mo.dehghani@sutech.ac.ir|'Department of Electrical Engineering, Sharif University of Technology, Tehran, Iran. ${ }^{2}$ Department of Electrical and Electronics Engineering, Shiraz University of Technology, Shiraz, Iran. 
The power control of the constant-speed wind turbines is performed mechanically. But, in the variablespeed ones, it is performed both mechanically and electrically. Therefore, the oscillation damping is expected to be carried out both mechanically and electrically. The DFIG wind turbines have two converters (one on the rotor side and the other on the grid side). The produced real and reactive powers are controlled using the rotorside converter. Applying some modifications on rotorside converter, the oscillations of the power system are able to be damped.

The mechanical damping controllers manipulate the pitch angle, so that the output power of the wind generator is changed and also it robusts to the power oscillation of the system. A fuzzy proportional-integral-derivative (PID) power system stabilizer is proposed in [2] that applies the grid frequency and the pitch angle as its inputs. In [3], an oscillation damping controller of the power system is presented based on the fuzzy logic considering the nonlinear aerodynamic parameters. The major drawback of the proposed controllers is that they are often utilized in the constant-speed wind generators and have a slow time response. On the other hand, the electrical damping controllers are usually implemented on the variable-speed wind generators.

In reference [4], a controller for the DFIG wind turbine is proposed to damp the power system oscillations. The controller works based on the flux and pitch control. Its main advantage over other generator stabilizers is that it does not affect the performance of the voltage control. Although the authors have demonstrated the effects of the damping controller on improving the oscillation suppression, they have only studied the local oscillations rather than the inter-area ones. The effects of increasing the wind power penetration level on the oscillations damping have been studied in reference [5], where it has been shown that DFIG has a general tendency to damp the oscillations. This is while the oscillation damping may decrease due to some specific penetration levels. A damping controller with a special structure is proposed in [6]. This controller applies the nonlinear multivariable optimization methods and uses the DFIG speed and the stator power as its inputs. The results indicate that the proposed controller reduces both the electromechanical oscillations of the power system and the torsional oscillations. The authors of the references $[7,8]$ showed that it is possible to considerably suppress the inter-area oscillations. It is possible by designing the DFIG controller using the root locus method. In long transmission lines, these outcomes become even more important. The relative differences between the rotor angles of two generators in two different areas are the inputs of the proposed controller. However, this controller only improves one oscillatory mode and for covering more modes, a more complicated controller is required.
The uncertainties of the system involve the modeling and linearization, and approximation errors in eliminating the high frequencies, the working point changes, variations in the wind speed, and the grid faults leading to the line disconnection. As a result, in the present work, the controller is designed using the robust control approaches. Here, the controller is designed using the frequency responses, so that the system exhibits an acceptable performance under the allowable variations of the uncertainty parameters. Also, it remains stable and has the nominal and robust stability together with the nominal and robust performance [3, 9]. In references [10, 11], a remote-control block and a dynamic stabilizer which improve dynamic oscillation damping have been proposed; however, in these studies, only effects of faults are considered and uncertainties including different operation points are not investigated. In paper [12], an auxiliary controller has been added to the active power control loop which is called the virtual inertial controller and improves the damping of between area oscillations in a system where the inertia of the machines is variable; but, does not take into account different operation points and events. Due to the fact that the inter-area modes are a result of the inter-area transmitted power, the input signal of the controller might be chosen so that it depends on the transmitted power (e.g. the generator speed, or the rotor angles with respect to each other). Then, the control signal works as an input to the DFIG control loop; consequently, the inter-area modes can be affected. The main contributions of the paper are summarized as bellow:

- Designing the auxiliary controller based on robust control on DFIG power control circuit to develop damping of inter-zone oscillations.

- Combining $\mathrm{H}_{2} / \mathrm{H}_{\infty}$ robust control approaches to design the controller in order to enhance the final and transient responses of the system.

- Creating a robust system to various disturbances using the controller design with LMI method and creating a hybrid objective function.

The rest of this paper is organized as follows: Section II discusses the DFIG modeling; Section III describes the proposed auxiliary robust controller. The presented system and the controller design are developed in Section IV. Finally, the conclusion is drawn in Section V.

\section{DFIG modeling}

Figure 1 depicts the schematic view of DFIG generators, where, the stator is directly connected to the grid, but the rotor is connected via the power electronic converters. These 
converters consist of two back-to-back pulse width modulation (PWM) converters; one on the rotor side (RSC) and the other on the grid side (GSC). Controlling the grid-side converters (GSCs) will result in controlling the $\mathrm{DC}$ voltage link and the input reactive power. The real and reactive powers produced by the turbine are controlled by the rotor-side converter (RSC). The model of the DFIG-based wind power stations consists of the models for the aerodynamics, speed control of the wind turbine, DFIG, machine dynamics, turbine shaft dynamics, DC link dynamics, and converters' control systems.

\subsection{DFIG generator model}

The dynamic equations of DFIG with a 3-phase transmission to the reference frame are presented in Eq. 1 and Eq. 2 [7].

$V_{d q s}=-r_{s} l_{d q s} \mp \omega_{s} \lambda_{q d s}+\frac{d}{d t} \lambda_{d q s}$

$V_{d q r}=-r_{r} l_{d q r} \mp\left(\omega_{s}-\omega_{r}\right) \omega_{s} \lambda_{q d r}+\frac{d}{d t} \lambda_{d q r}$

where, $I, v, i$ and $r$ denote the flux linkage, voltage, current, and resistance, respectively. $\omega_{s}$ and $\omega_{r}$ are the angular velocities of the synchronous reference frame and the rotor, respectively.

\subsection{Turbine model}

The dynamics of the turbine's driving parts is presented by the two-mass model in the stability studies as follows (the turbine is connected to the generator via a gear box):

$2 H_{t} \frac{d \omega_{t}}{d t}=T_{m}-k \theta_{t w}-c \frac{d \theta_{t w}}{d t}$
$2 H_{r} \frac{d \omega_{r}}{d t}=-T_{e}+k \theta_{t w}+c \frac{d \theta_{t w}}{d t}$

$\frac{1}{\omega_{e}} \frac{d \theta_{t w}}{d t}=\omega_{t}-\omega_{r}$

$T_{e}=L_{m}\left(I_{q r} I_{d s}-I_{q s} I_{d r}\right)$

where, $H_{t}$ and $H_{r}$ represent the inertia of the turbine and generator, respectively. $\omega_{t}$ is the turbine's angular velocity, $\omega_{e}$ shows the base electrical angular velocity, $\theta_{t w}$ designates the shaft's torsion angle; finally, $k$ and $c$ are the stiffness and damping coefficients, respectively.

A turbine converts the wind kinetic energy into the mechanical energy. The wind turbine mechanical torque is calculated as follows.

$T_{m}=\frac{\rho \pi D^{2} C_{p} U_{w}^{3}}{8 \omega_{t}}$

where, $\rho$ is the air density, $D$ denotes the rotor diameter, $C_{p}$ represents the wind turbine power coefficient as presented in Eq. 5 (it indicates how the wind energy affects the turbine) [13], and $U_{w}$ denotes the wind speed. Figures 2 and 3 show pitch angle control and RSC control, respectively.

$C_{p}=0.22 *\left(\frac{116}{a}-0.4 \beta-5\right) * \exp \left(-\frac{12}{a}\right) ; \alpha=\frac{D \omega_{t}}{2 U_{w}}$

where, $\beta$ is the pitch angle and $\alpha$ is a definition parameter that is defined in Eq. 5.

\subsection{Control model of converters}

If the terminal voltage of the generator is rotated on new coordinates so that all the voltage lies on the $q$ axis
Fig. 1 A schematic view of DFIG

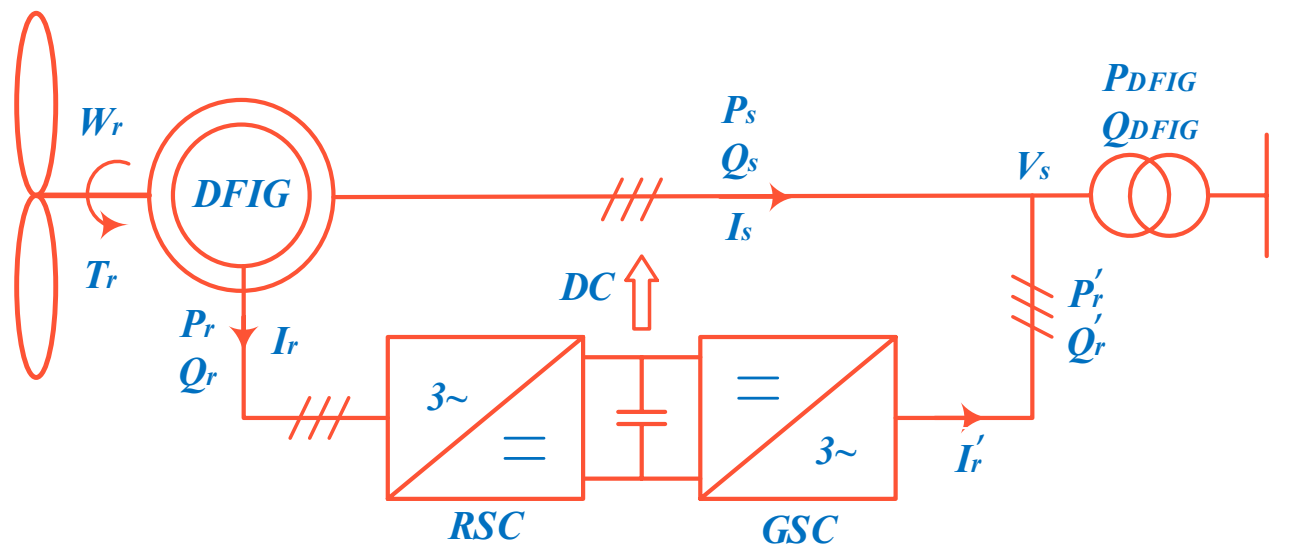

SN Applied Sciences 
Fig. 2 Pitch angle control

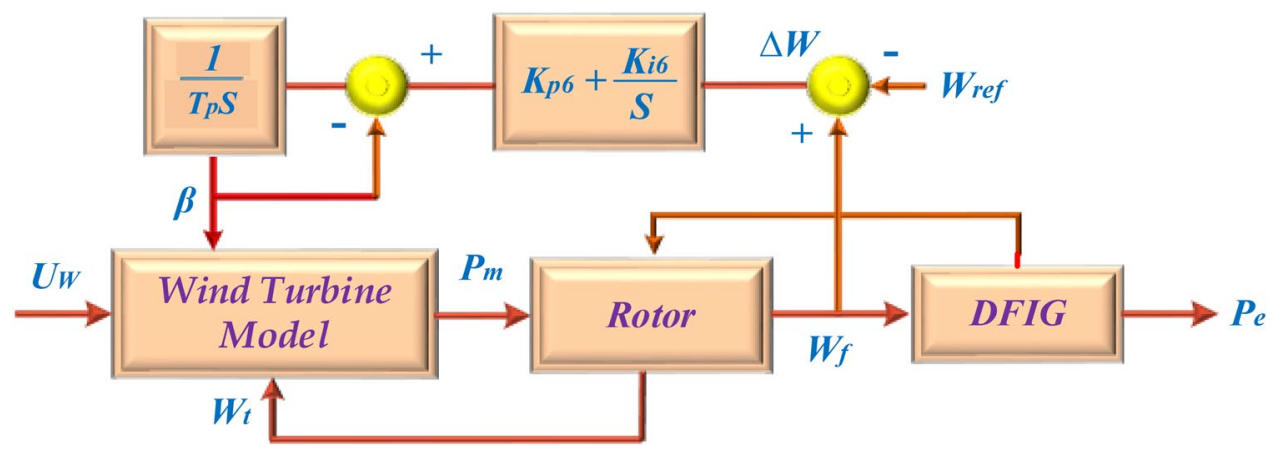

Fig. 3 RSC Control

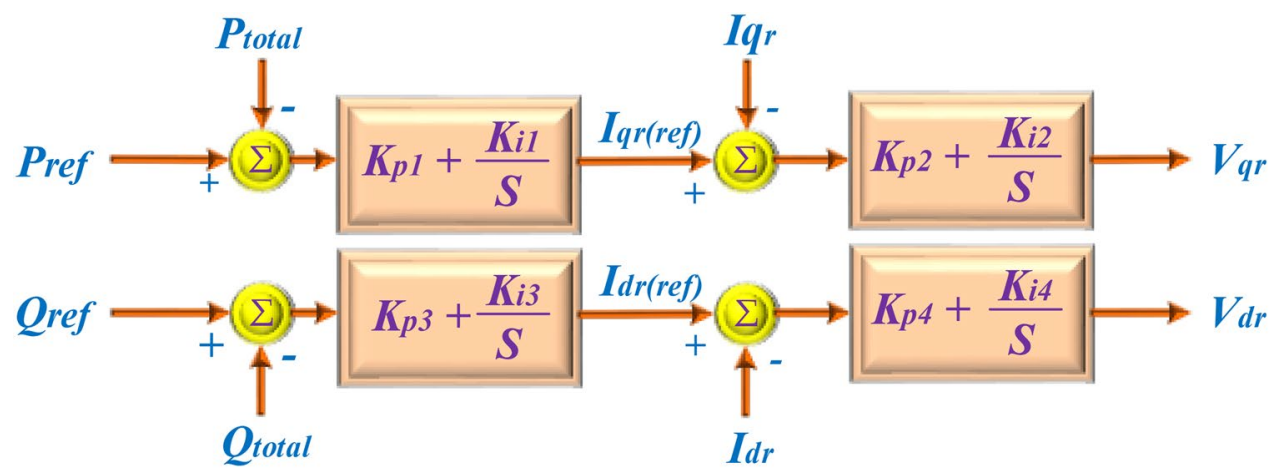

$\left(V_{d}=0\right)$, then the active power relies on $I_{q}$ and the reactive power depends on $I_{d}$ [13]. The RSC controls the rotor voltage so that the active and reactive power of DFIG are controlled. As can be seen from the Fig. 3, two PI controllers are employed to make the DFIG power comparable with the reference power. They also specify the reference current for the rotor. The rotor current is then compared to the reference current, after that, the rotor voltage is determined by a PI controller.

The grid-side converter should allow the rotor active power and the RSC power to be passed through it, so that the DC link voltage remains unchanged. The reactive power of this converter is controllable and it is usually assumed to be zero in order to reduce the costs and the converter volume.

In the following sections, the robust control and $\mathrm{H}_{2} / \mathrm{H}_{\infty}$ method will be presented, and based on them, a controller will be proposed.

\section{Using $\mathrm{H}_{2} / \mathrm{H}_{\infty}$ Robust control in DFIG to enhance system oscillation}

Maintaining and improving the system stability against the modeling errors and uncertainties is the main objective of the robust control [14]. A robust controller should guarantee the stability and performance of a system under all states and uncertainties. These are the requirements for a closed-loop system known as the robust stability and the robust performance. The authors of reference [14] discussed the relationship between $H_{\infty}$ and the stability. The $H_{\infty}$ method employs a proper weight function and it is designed in the frequency domain; while the $\mathrm{H}_{2}$ design method improves the system performance in the time domain (i.e., it deals with the transient behavior of the system) [15]. In the past decades, the power system stabilizers were designed based on the robust control principles, only deal with the modeling error and uncertainties and they are single-objective. In the real-world systems; however, the $H_{\infty}$ controller cannot provide all designing specifications like the noise or random errors as the $\mathrm{H}_{2}$ controller does. On the other hand, the $\mathrm{H}_{2}$ controllers do not guarantee some features like the robust stability and performance. As a result, the present work tries to combine these methods, so that their advantages could be utilized [15]. The controller input is the generator rotor speed difference between two areas and the controller output apply to the active and reactive power references of DFIG.

\subsection{The proposed damping controller}

The multi-objective configuration of the damping controller for the power system stabilization is proposed in Fig. 4.

$G_{0}(s)$ is the system state space model and $K(s)$ is the controller state space model. In fact, norm 2 represents the signal energy, and infinite norm indicates the gain of 
the system or its stability. For this reason, a combination of the two has been used in the design of the controller. The method of obtaining the system parameters is based on the equations of state space and linearization around the working point and $G_{0}(s)$ has been obtained.

Regarding the controller design, the components of matrix A of the controller mode space are defined as the optimization variable; then, it is optimized using the objective function mentioned in Eq. 6, and the optimal controller is obtained which means that, the controller is defined with the initial variables and the outputs are obtained; then, the norm 2 and their infinity are calculated, and the corresponding value is calculated according to Eq. 6. In the same way, the best answer will be found with the LMI optimization algorithm.

As can be seen from the Fig. 4 , the output channels of $Z_{\infty}$ and $Z_{2}$ are associated with the $H_{\infty}$ and $H_{2}$ performances, respectively. $W_{1}(s)$ is a low-pass filter on the $H_{2}$ output channel. This filter aims to enhance the system's transient behavior. $W_{2}(s)$ is a high-pass filter or a small constant value on the output channel of the $\mathrm{H}_{2}$ controller and its purpose is to minimize the noise effect on the controller's output. $\mathrm{W}_{3}(\mathrm{~s})$ is a high-pass filter on the $H_{\infty}$ controller's output. It tries to maintain the system stability under the various uncertainties. All weights are chosen based on the system nature. In other words, they are acquired empirically or by several trail-and-errors.

\subsection{Linear matrix inequality (LMI) method in designing a hybrid Robust controller considering the pole placement zone}

The LMI method is recently proposed to solve the $H_{\infty}$ problem. The numerical analyses with the LMI formulation provide several advantages. They prevent the pole or zero elimination and accept different constraints for different objectives [16]. This technique has also been employed to design the power system damping controllers in [2]. The LMI method in designing the hybrid $\mathrm{H}_{2} / \mathrm{H}_{\infty}$ controllers with the distinct poles was explained in [4]. Figure 5 shows a closed-loop system with a controller. It is assumed that $T_{2}(s)$ and $T_{\infty}(s)$ are the closed-loop transformation functions from $\omega$ to $Z_{2}$ and $Z_{\infty}$, respectively. $\omega$ in Fig. 5 represents the speed difference of the generators.

Now, the multi-objective problem of the controller design with an output feedback is emerged to satisfy the following qualities:

- The infinity norm of $T_{\infty}(s)$ (the gain) should be held smaller than the pre-determined value such that the robust stability and performance are obtained.

- The 2-norm of $\mathrm{T}_{2}(\mathrm{~s})$ has to be lower than a pre-specified value to improve the system's transient behavior.

- The cost function of Eq. 6 should be minimized and its terms have to be weighted with respect to their significance.

$\alpha\left\|T_{\infty}\right\|_{\infty}^{2}+\beta\left\|T_{2}\right\|_{2}^{2}$

- $\alpha$ and $\beta$ are the coefficients that specify the significance of each objective.

- The poles are to be placed at the proper locations. When the closed-loop poles are in the specified area, it ensures the minimum damping ratio, the minimum rate of decay, and acceptable controller gains. In this

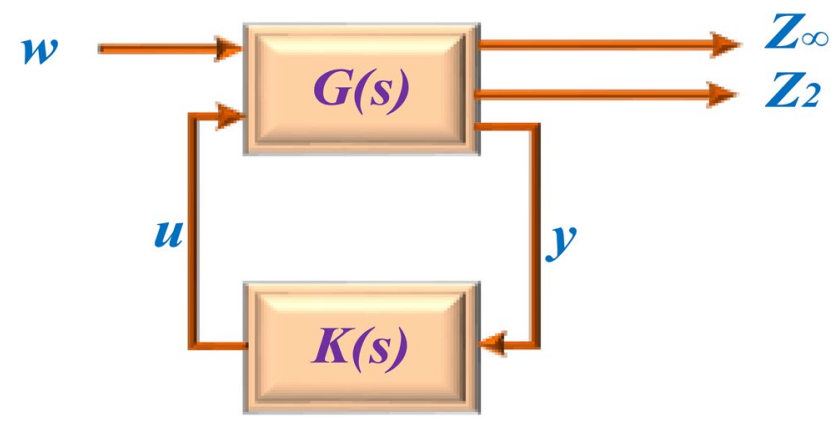

Fig. 5 A closed-loop system with a controller

Fig. 4 The proposed multiobjective controller

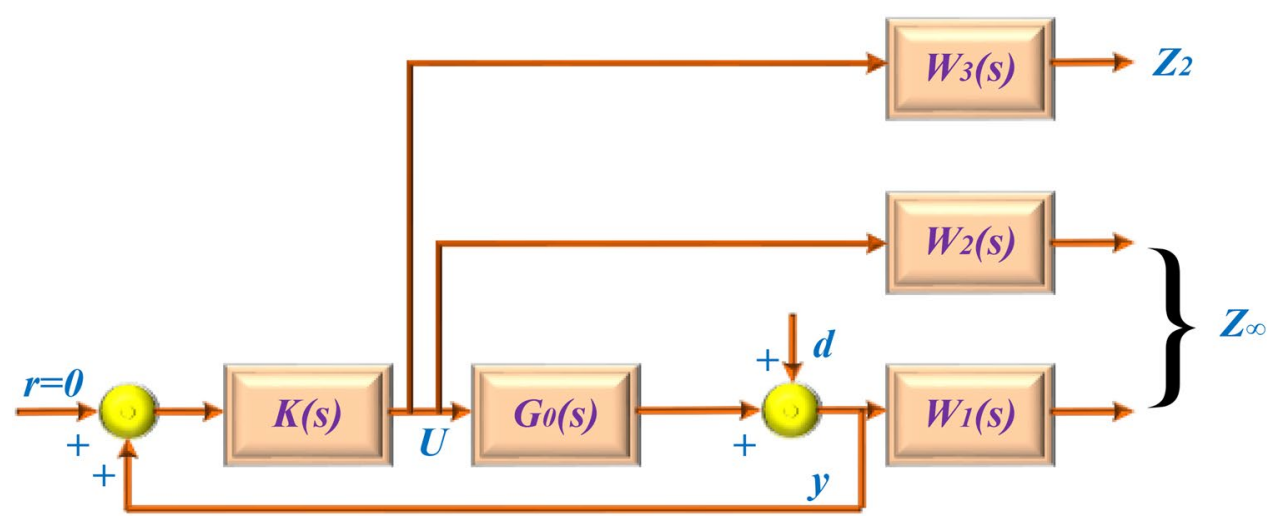


case, the transient response of the system is improved. While placing the poles, other control objectives (such as the robust performance, robust stability, overshoot level, rise time and so on) should also be satisfied [17].

If $z$ denotes the closed-loop poles, then the pole placement should be done under the following constraint. This constraint is applied when the objective function is being optimized $(r e(z)<-\delta)$ : "re" is real part of the closed-loop poles.

$z+\bar{z}+2 \delta<0$

\section{Controller design and simulation}

The solution method of the proposed model is as follows: 1- Appling power flow and obtaining the steady state working points for all state variables. 2- Linearization of the system around the specified working point and reduction of the system order. 3- Designing the auxiliary controller based on the robust control. 4- Implementation of the controller on the main nonlinear system. 5 - The test of the system with the designed auxiliary controller and without the controller in different working points and events.

From Fig. 6, the simulations are carried out for a 2-area system with four machines. The grid specifications are presented in [18]. This grid consists of two areas; each with two generators. The produced power of each synchronous generator is about $700 \mathrm{MW}$. The synchronous generators are assumed to be ideal and equipped with the Automatic Voltage Regulator (AVR). Their model is nonlinear and third-order; it does not consider the transformer voltages.
This model is usually employed in the simplified stabilizing studies [19].

In order to confirm the effectiveness of the proposed controller, we assume that no station possesses PSS. A DFIG-based wind farm is connected to the grid via a $10 \mathrm{~km}$ transmission line. The parameters for DFIG and its control system are presented in [20]. Normally, the wind farm supplies a power of $150 \mathrm{MW}$ to the grid, and nearly $400 \mathrm{MW}$ is transmitted from the area 1 to the area 2 .

\subsection{Linearization at the working point}

In this part, the simulations are performed assuming that the generators 1 and 3 adjust their bus voltages as 1.03 per unit (p.u.) and the generators 2 and 4 are tuned with 1.01 p.u. The DFIG reference active power is $150 \mathrm{MW}$ and the reference reactive power is considered to be zero.

Assuming two inputs of the active and reactive reference power for DFIG and one output of the angular velocity difference in the generators 2 and 3 , the linearization is performed in the MATLAB software. The effects of the oscillatory modes on the state variables are studied given the system state matrix and the contribution factor. The results for the electromechanical oscillatory modes with no controller are given in Table 1.

First, the system is linearized around the operating point, then by calculating the system state matrix, eigenvalues and their eigenvectors are calculated, and consequently, by calculating the participation coefficients, the degree of participation of state variables in eigenvalues (system oscillation modes) is investigated. The low frequency oscillation mode in which the generator speed of one area and the generator speed of the other area are more involved, is the interregional oscillation mode. The

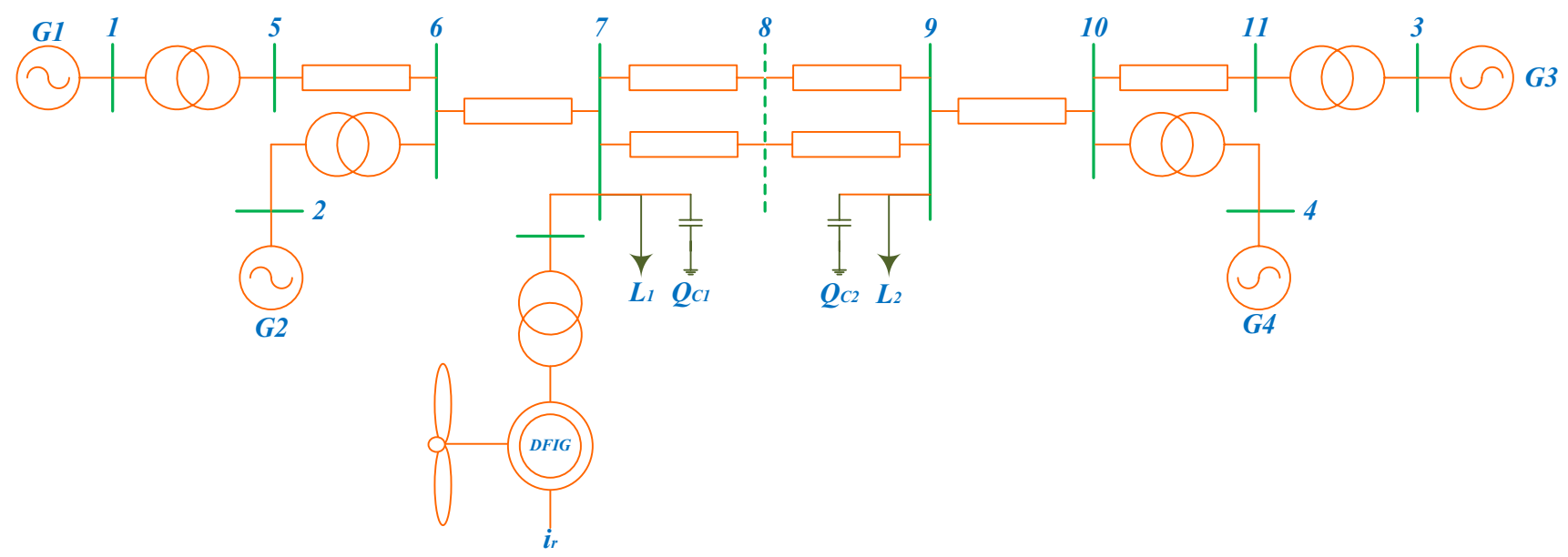

Fig. 6 The proposed case study 
participation coefficients are presented in the reference [19].

In the system state matrix, $\lambda_{i}$ are the system eigenvalues, $x_{i}$ are the system state variables, $\varphi_{i}$ are the right eigenvectors of the state variables, $\varphi_{i}$ are the left eigenvectors of the state variables, and $a_{k j}$ are the row element $k$ and the $j$ column of the matrix $A$. In this regard, we have:

$A \varphi_{i}=\lambda_{i} \varphi_{i}$

$\frac{d A}{d a_{k j}} \varphi_{i}+A \frac{d \varphi_{i}}{d a_{k j}}=\frac{d \lambda i}{d a_{k j}} \varphi_{i}+\lambda_{i} \frac{d \varphi_{i}}{d a_{k j}}$

Since $\phi_{i} \varphi_{i}=1$ and $\phi_{i}\left(A-\lambda_{i} l\right)=0$, Eq. 8 will be simplified as follow

$\frac{d A}{d a_{k j}} \varphi_{i}=\frac{d \lambda i}{d a_{k j}} \varphi_{i}$

$\left\{\begin{array}{l}\frac{d A_{m n}}{d a_{k j}}=0 m n \neq k j \\ \frac{d A_{m n}}{d a_{k j}}=1 m n=k j\end{array} \Rightarrow \frac{d \lambda i}{d a_{k j}}=\phi_{i k} \varphi_{j i}\right.$

Now, by forming the above matrix, we can analyze the degree of participation of each of the state variables in each oscillation mode.

The results show the negligible damping in the electromechanical oscillations. This can be attributed to the zero $K_{D}$ and absence of PSS. The oscillatory mode of the pitch control has a low frequency. Since there is a considerable damping in this mode, its low frequency causes no major problem. The main objective of this paper is to increase the inter-area oscillatory mode damping.

\subsection{Design of the auxiliary robust controller}

It is hard to design a controller for the high-order systems. In order to do this, the order of the system is reduced. Order reduction is performed via the MATLAB software. First, the contribution of the modes that have a noticeable influence on the output, is measured in MATLAB. These modes have a considerable energy. In the present work, the order of the system is reduced from 31 to 6 . At last, the controller is implemented on the intact nonlinear system.

Table 1 Electromechanical oscillatory modes without a controller

\begin{tabular}{llll}
\hline Mode & Eigenvalue & Damping ratio & Frequency \\
\hline Inter-area & $2.839 \mathrm{j}-0.0408$ & 144 & 0.4518 \\
Area 1 & $5.612 \mathrm{j}-0.0654$ & 1.116 & 0.8932 \\
Area 2 & $6.498 \mathrm{j}-0.142$ & 2.19 & 1.034 \\
\hline
\end{tabular}

Section III presented the detailed specifications of the design. $\alpha$ and $\beta$ are chosen to be 0.9 and 0.1 , respectively. $\alpha$ is larger than $\beta$ due to the significance of the robust performance and control. Note that the model of system is approximated and has numeral uncertainties. Due to the fact that the controller should exhibit an appropriate performance under all conditions, the robust performance and stability seems to be more important.

As previously explained in the Section III, the weight factors are specified via the numeral simulations and trialand-errors. The suggested values for the weight factors are as follows.

$\omega_{1}=\frac{1}{S+10}, \omega_{2}=10^{-7}, \omega_{3}=\frac{S}{S+80}$

where, $S$ represents the Laplace variable.

The transient pole zone should be specified so that the transient behavior of the system is enhanced and the robust performance is maintained. According to the simulations, the left side of the imaginary axis, $X=-0.4$, was determined as the pole placement zone (i.e., the damping is more than 0.4).

According to the Section III, the controller is implemented on the system with the unknown state space parameter and the suggested configuration. Using the information presented in the previous section, the unknown parameters are obtained by the LMI optimization algorithm embedded in the MATLAB software. Once the controller is designed and the simulations are carried out, the state space parameters of the controller are specified. The resulting controller has the order Eq. 11 which is considerably high for such a controller. Therefore, considering the system order, its order will be reduced to Eq. 6 . The state matrices of the controller are presented in Eqs. (11-14).

After distributing the initial load and linearizing the system around the operating point, the system state equations are extracted. Then, using the LMI linear optimization method [21] and given the objective function of Eq. 6, also considering the parameters of the proposed controller state matrices as optimization variables, the output of the optimization is described in the following matrices.

$A_{K}=\left[\begin{array}{cccccc}-0.1227 & -6.152 & -1.952 & 8.644 & 386.74 & 0.508 \\ -8.046 & -299.5 & -85.088 & 325.78 & 28643 & 110.39 \\ -2.96 & -104.6 & -30.9 & 117.8 & 10106.4 & 39.6 \\ 12.07 & 548.6 & 148.5 & -624.01 & -52485 & -195.6 \\ 2000 & 7710 & 21225 & -87830 & -7432590 & -27980 \\ 1.493 & 39.79 & 11.97 & -37.2 & -4418 & -64.54\end{array}\right]$

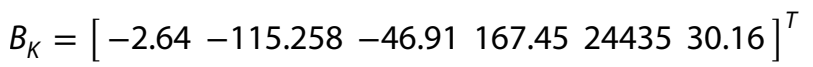


$C_{K}=\left[\begin{array}{cccccc}301.5 & 187.3 & -53.7 & -84.8 & -7622 & -31.3 \\ -150.4 & -116.1 & 122.8 & 1.43 & 3404 & -15.7\end{array}\right]$

$D_{K}=[-3.049-2.74]^{T}$

\subsection{The effects of the controller on the oscillatory modes of the linearized system}

After applying the designed controller to the main system, the system is linearized again around the working point and local and interregional oscillation modes are identified using the participation coefficients. Table 2 shows the controller effects on damping the electromechanical oscillations. The results show a 22-time larger damping in the inter-area oscillatory modes. The local oscillation mode of the area in which DFIG is located has a damping ratio of 14 times larger than the uncontrolled case, but that of the farther area remains approximately unchanged. It is possible to make the oscillation damping even more, but it degrades the system robust performance against unwanted errors. The main reason due to which the controller has been incapable of affecting the local oscillation

Table 2 The electromechanical oscillatory modes in the presence of a controller

\begin{tabular}{lccl}
\hline Mode & Eigenvalue & Damping ratio & Frequency \\
\hline Inter-area & $2.8 \mathrm{j}-0.64$ & 22.86 & 0.45 \\
Area 1 & $5.56 \mathrm{j}-0.81$ & 14.54 & 0.892 \\
Area 2 & $6.5 \mathrm{j}-0.144$ & 2.2 & 1.034 \\
\hline
\end{tabular}

damping of the farther area is that the aforementioned local oscillation occurs in the area 2 and it is not either observable or controllable from the area 1 where DFIG exists. In the next sections, the performance of the controller is investigated under the high-amplitude disturbances.

\subsection{Performance of the controller in the time-based simulations under different conditions}

In this section, different working points and disturbances are applied to the system and the controller performance is evaluated in the time-domain simulations. Several alphabetically ordered scenarios (from $a$ to $e$ ) are defined as follows:

$a$ - In this case, the system works at the normal working point; then, the controller performance is observed when a short circuit happens for $0.1 \mathrm{~s}$ in the bus 5 . The angular and rotational speed differences of the generators 2 and 3 are depicted in Fig. 7, where, the controller has an acceptable performance. Figures 8, 9 and 10 illustrate the active and reactive power of DFIG, $d$ - and $q$-axis voltages applied to the rotor, and the pitch angle of the DFIG turbine, respectively. The figures reveal that DFIG has to change its output active and reactive power in order to reduce the inter-area and local oscillations. On the other hand, the rotor voltage should be controlled so that the desired power is tracked; this can also be done by manipulating the pitch angle. The simulations show that DFIG utilizes both the rotor voltage and the pitch angle to reduce the system oscillations.

Figures $7,8,9$ and 10 are related to the simulation results of case (a). As shown in the Fig. 7, the attenuation of the speed difference and rotor angle difference signals of the generators 2 and 3 contain the oscillating modes of the
Fig. 7 Angular and rotational speed differences in the simulation Case $a$
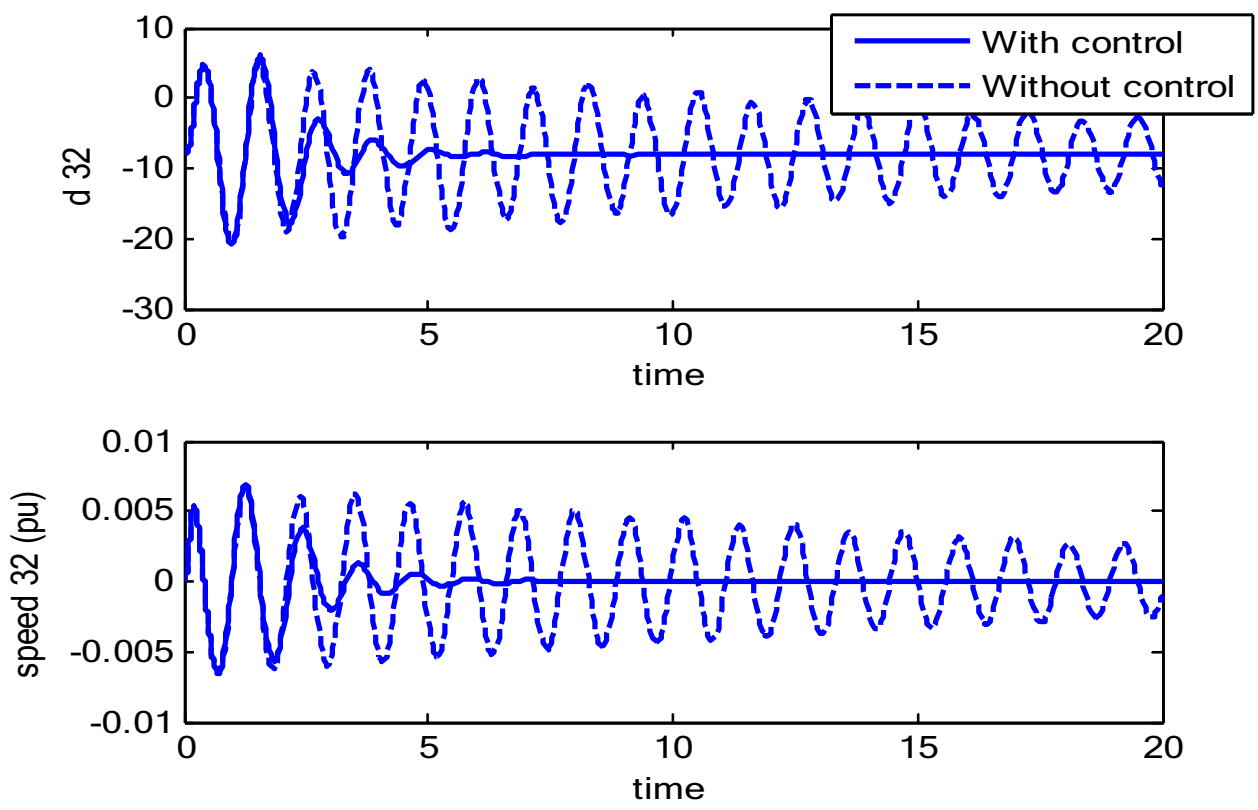
Fig. 8 DFIG active and reactive power in the simulation Case $a$
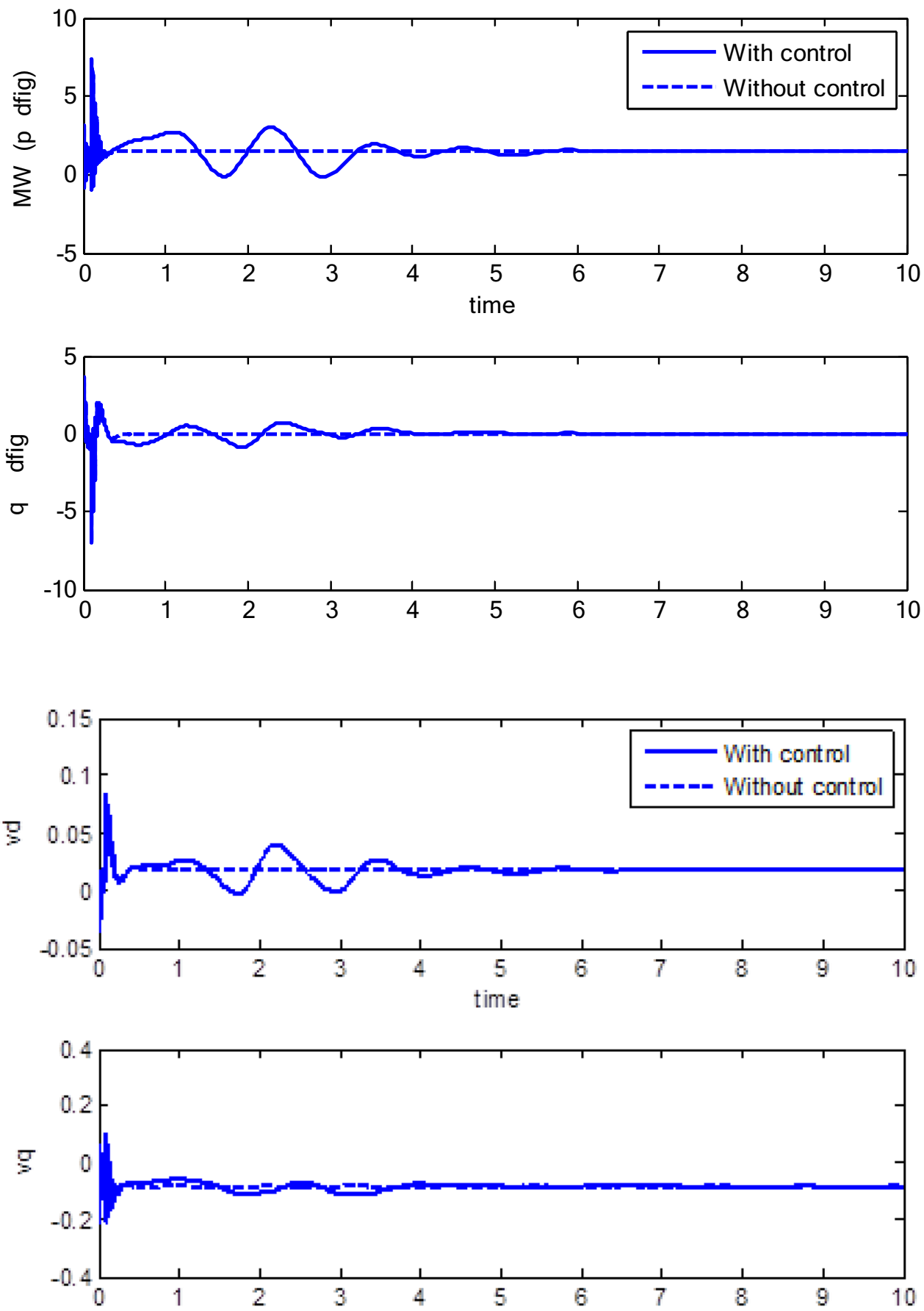

system without the auxiliaries controller that are high, and the dampers are slowly died out, but these oscillation modes are increased and died out rapidly because of auxiliary controller which is added. Figure 7 is proved the performance of the auxiliary controller.

As can be seen from the Fig. 7, the oscillations of the rotor angle difference between two generators in two different regions, which represent the same inter-region mode fluctuations, In the case where the auxiliary controller is not added, it is still not damped after $15 \mathrm{~s}$, but with the addition of the auxiliary controller, these oscillations are completely damped after $5 \mathrm{~s}$. Figure 8 shows the DFIG's active and reactive power in the simulation case $a$.

As can be seen from the Fig. 8, the input signal of the DFIG's active and reactive power adjustment control loops is a constant signal against disturbances in the absence of auxiliary controller. When, there is an auxiliary controller, if any disturbances are entered to the system, DFIG power controller input signals are mixed with the auxiliary controller output signals. As a result, 
Fig. 10 The rotor speed and the pitch angle in the Case $a$
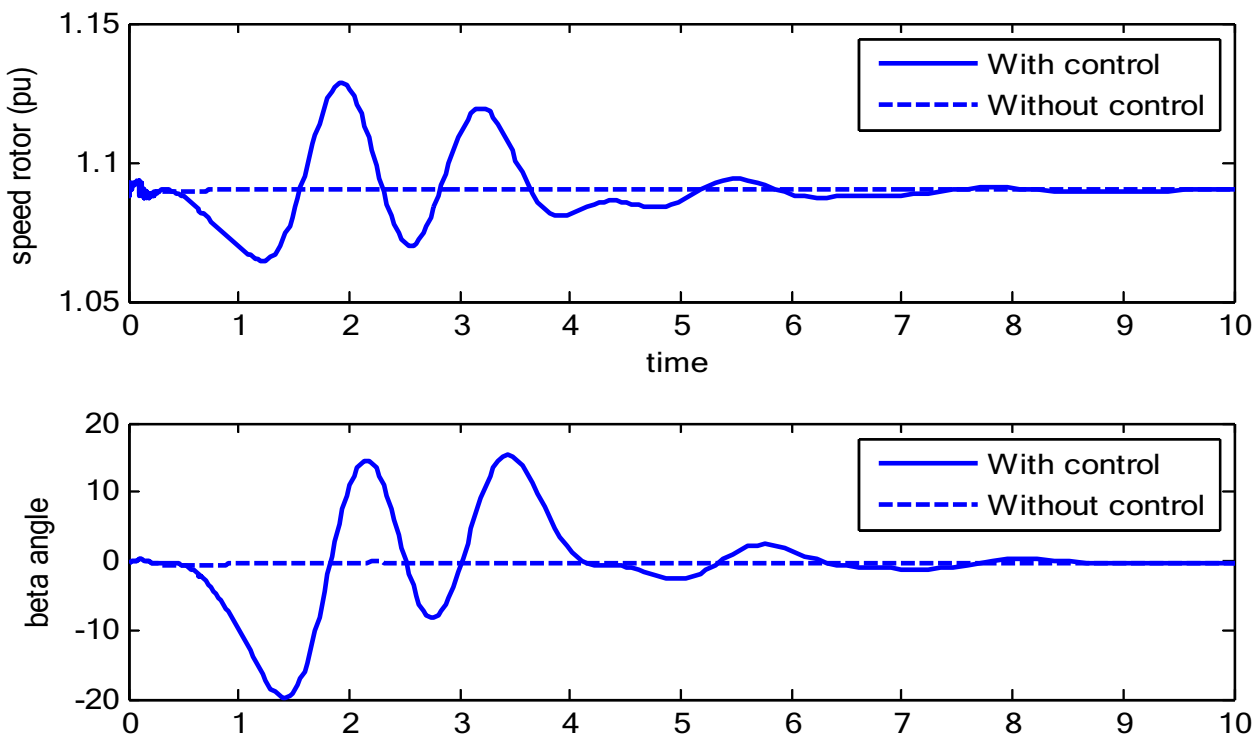

the damping oscillation is improved as well by changing the active and reactive power temporarily. Figure 9 displays the rotor voltage (p.u.) in the case $a$, and Fig. 10 depicts the rotor speed and the pitch angle in the case $a$.

The input changes of the active and reactive power of the control loops that are created by the proposed auxiliary controller against disturbances, are made in two ways by DFIG. As shown in the Fig. 9, part of the power is compensated by the inverter and converter in the rotor side with voltage variations. Another part of the power is generated by the torsion angles variation of the turbine blades as can be seen in the Fig. 10 .
Figures $11,12,13,14$ are shown the oscillation of speed difference and rotor angle difference of generators 2 and 3 against different types of disturbances. As can be seen, the auxiliary controller is increased the damping between the zones and even, the unstable system in the simulation of scenario $e$ has been stabilized by addition the auxiliary controller and the unstable poles of the system are within the specified polarization range.

$b$-The system operates at its normal working point, but the wind speed becomes 10 times larger for a period of $2 \mathrm{~s}$.

$c$-One line between the buses 7 and 8 is disconnected and the system undergoes a short circuit problem in the bus 5 for $0.1 \mathrm{~s}$.
Fig. 11 Angular and speed differences for the generators 2 and 3 in the simulation Case $b$

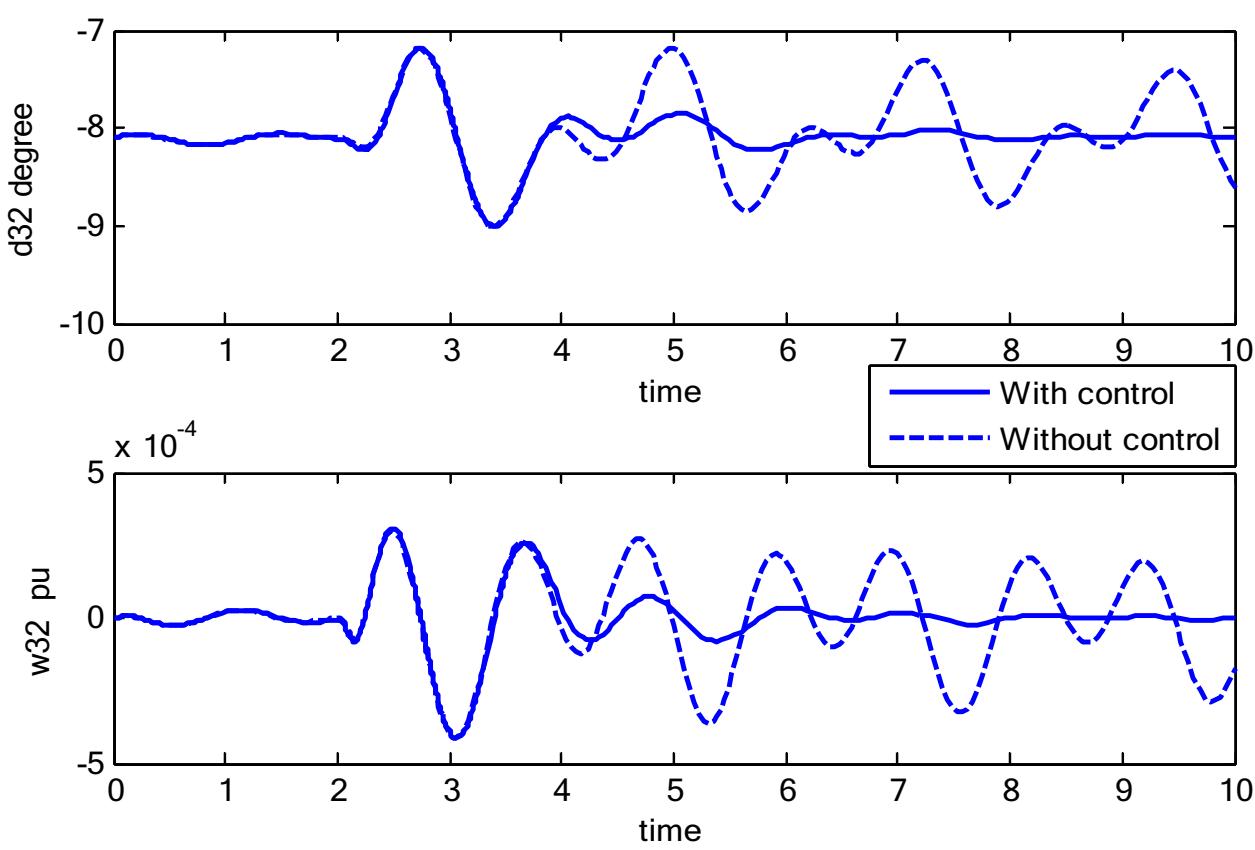


Fig. 12 Angular and speed differences for the generators 2 and 3 in the simulation Case $c$
Fig. 13 Angular and speed differences for the generators 2 and 3 in the simulation Case $d$
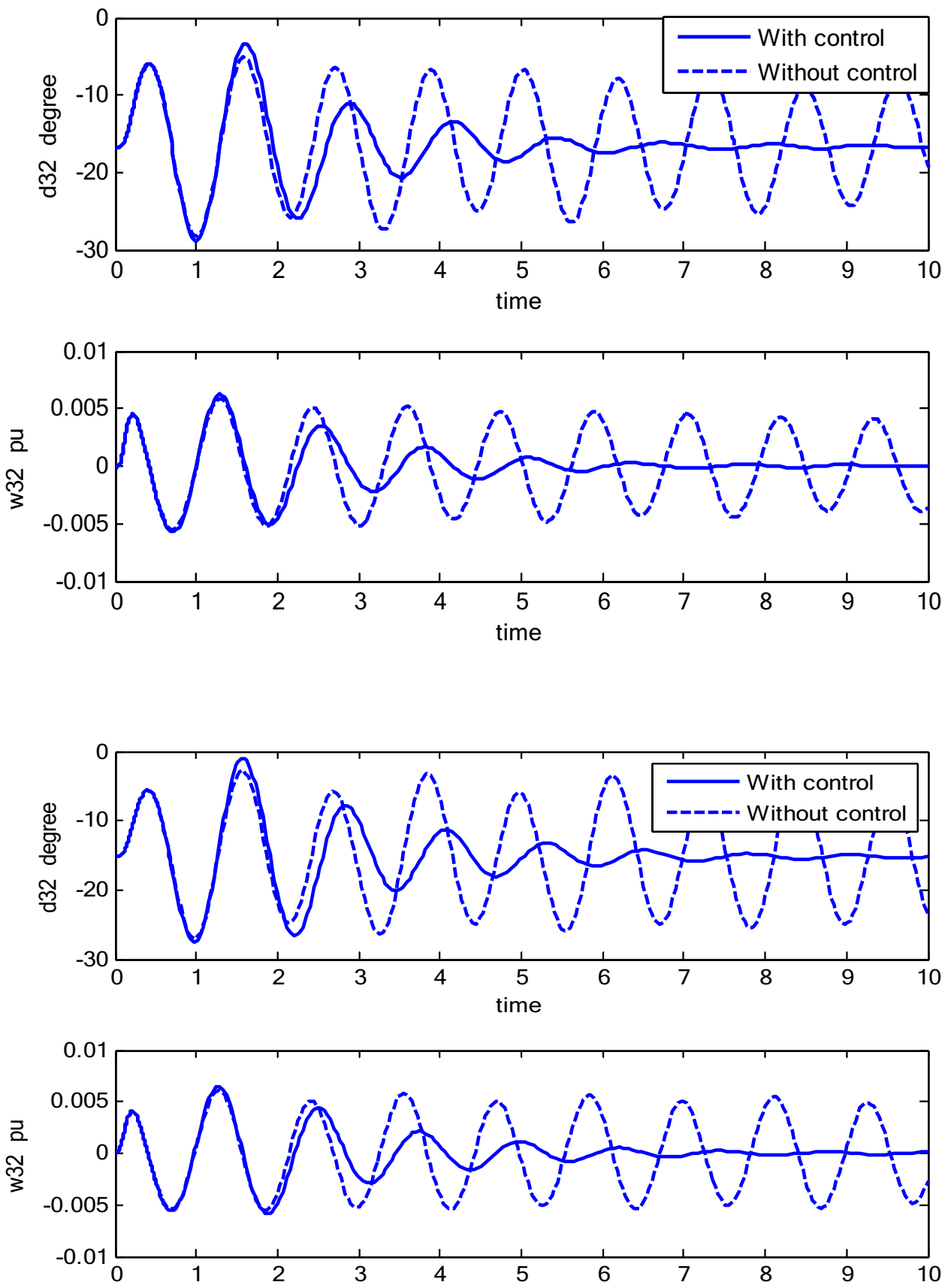

$d$-The active power of the load 2 is increased by $150 \mathrm{MW}$ and a short circuit occurs in the bus 5 for $0.1 \mathrm{~s}$.

$e$-The load 2 is increased by $150 \mathrm{MW}$ and the power arrangement of the generators gets changed (the power of generators 2 and 3 is increased by $100 \mathrm{MW}$ and that of the generator 4 is decreased by $100 \mathrm{MW}$ ). This implies an uncertainty in the working point. Then, the wind speed gets 0.2 times of its previous amount; also, the DFIG's active and reactive power become zero (the uncertainty in the wind speed and DFIG power). In addition, a short circuit problem occurs in the bus 5 for $0.1 \mathrm{~s}$.
The angular (in degrees) and the speed differences (p.u.) between the areas are illustrated in Figs. 11,12, 13 and 14.

In all simulation cases, the controller has an acceptable performance in the presence of various uncertainties. Also, in the simulation Case $e$, the controller stabilizes the instable system. 
Fig. 14 Angular and speed differences for the generators 2 and 3 in the simulation Case $e$
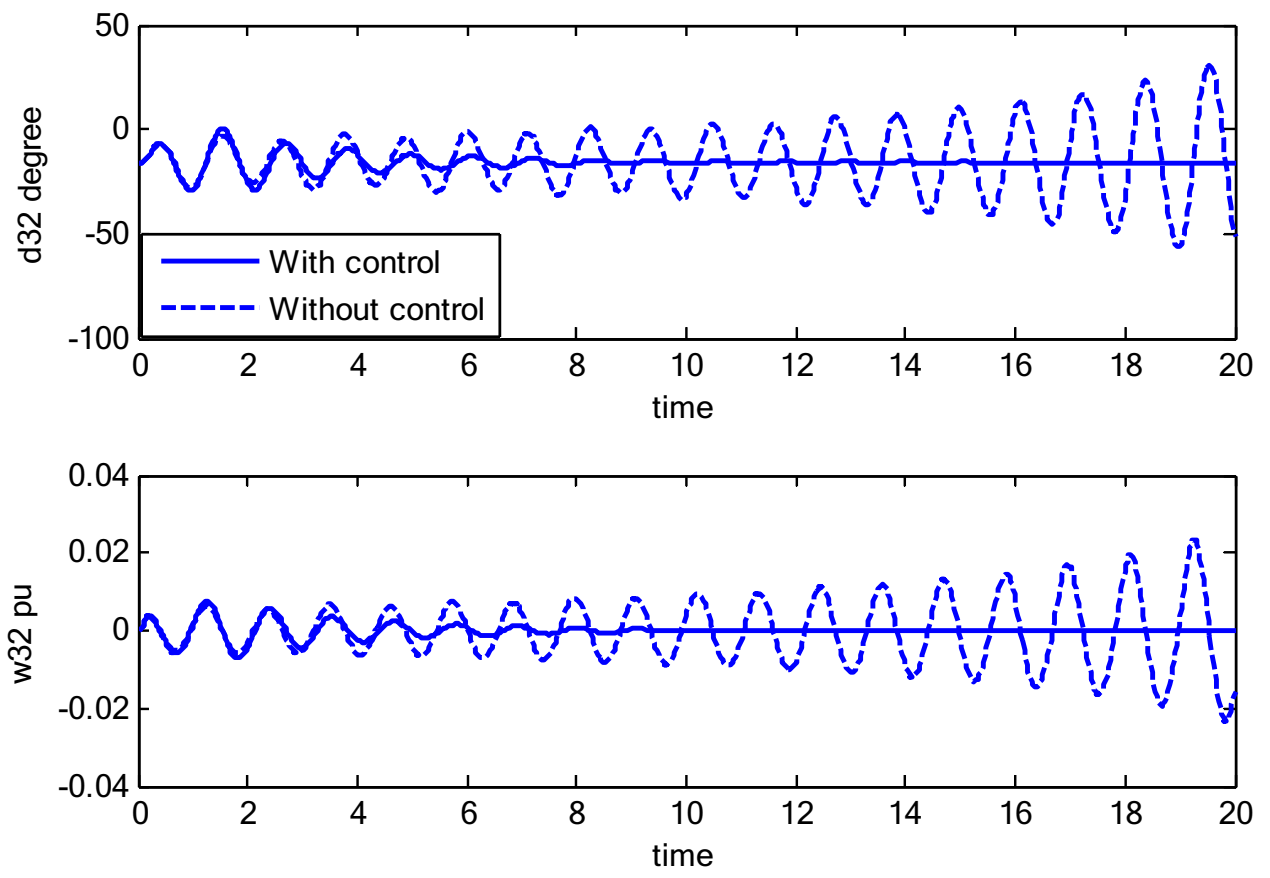

\section{Discussion}

In this research, the proposed method and the designed controller have the ability to function properly and quickly against various system disturbances. The simulated scenarios confirmed that the implementation of the proposed controller has sufficient efficiency and performance against changes in the operating point and various disturbances in the system.

The methods presented in recent papers are based on the geometric location [8] of the poles or fuzzy control [3, 17], which only consider improving the damping of interregional oscillations, and do not guarantee the proper performance of the designed controller in different operating conditions and turbulence. Whereas, the proposed controller considering $H_{\infty}$ to enhance stability and $\mathrm{H}_{2}$ to improve transient response, which given both appropriate velocity and performance against perturbations.

In [22], the main focus of the controller design was on the speed of the auxiliary controller; however, the efficiency and robustness of the controller in various operating conditions and disturbances have not been investigated. But in this paper, the proposed approach has been considered and simulated in various scenarios. In [23], the design of the controller was based on the improvement of the location of eigenvalues (Root Locus), which this method did not have the proper performance in different working points and disturbances. Also in [24], the controller was designed as a parallel two-channel, which has two major problems, first one, it slows down the performance, and the other is that it is not able to stabilize the unstable system, which in turn, it cannot to function properly at different working points. In [25], the designed controller was slightly more stable against disturbances; however, there was no discussion on the performance of the auxiliary controller at various working points.

\section{Conclusion}

In this paper, an auxiliary controller for DFIG based on robust control was designed based on the $\mathrm{H}_{2} / \mathrm{H}_{\infty}$ hybrid control method. Compared to other methods of controller design in recent research, the proposed method has the advantages of improving transient behavior and better damping of inter-regional fluctuations and having efficiency, robustness and stability to various uncertainties in the system. Also, in the proposed method, uncertainties, due to change of operating point and variety in wind speed and network changes, are considered. After applying the designed controller, its optimal effect on the system poles related to electromechanical oscillations was investigated. The results illustrated that this damping controller increased the inter-region oscillations by almost 22 times and the local oscillation damping by approximately 16 times the local generators. In addition to quenching inter-zone oscillations, this means that the wind generator with auxiliary controller can also suppress the local oscillations of the generators around it. The main idea of this controller is to use both active and reactive power modulation by a controller with two outputs that affects the DFIG electrical control. The proposed method improved the 
damping of fluctuations with appropriate changes in the rotor voltage, even if DFIG is not able to produce active power. One of the issues that can be considered in future research is to work on designing an auxiliary controller using DFIG controller to improve the transient stability of the system, or designing the same controller for other types of wind generators. Furthermore, the efficiency of the suggested method can be implemented on a real-time "hardware experiment" such as DSP/FPGA boards in the future works.

Acknowledgements We would like to thank Professor Taher Niknam from Shiraz University of Technology for his useful guidance in the various phases of preparing this research to improve the overall scientific quality of the paper.

\section{Compliance with ethical standards}

Conflict of interest The authors declare that they have no conflict of interest.

Open Access This article is licensed under a Creative Commons Attribution 4.0 International License, which permits use, sharing, adaptation, distribution and reproduction in any medium or format, as long as you give appropriate credit to the original author(s) and the source, provide a link to the Creative Commons licence, and indicate if changes were made. The images or other third party material in this article are included in the article's Creative Commons licence, unless indicated otherwise in a credit line to the material. If material is not included in the article's Creative Commons licence and your intended use is not permitted by statutory regulation or exceeds the permitted use, you will need to obtain permission directly from the copyright holder. To view a copy of this licence, visit http://creativecommons .org/licenses/by/4.0/.

\section{Appendix}

DFIG specifications (pu) are as follows.

$r_{s}=0.00488, r_{r}=0.00549, x_{m}=3.9530, x_{s s}=4.045, x_{r r}=4.052$

$H_{t}=4.33 s, H_{r}=0.62 s, k=1.11, c=1.5, T_{p}=0.25, k_{p 6}=700$

$k_{i 6}=500, k_{p 1}=0.6, k_{p 2}=0.1, k_{p 3}=0.012 ; k_{p 4}=0.1 ; k_{i 1}=3.2$

$k_{i 2}=10, k_{i 3}=0.3, k_{i 4}=2, v_{\text {base }}=35 \mathrm{kv}, S_{\text {base }}=150 \mathrm{MW}$

\section{References}

1. Gao B, Yunting Hu, Song R, Li R, Zhang X, Yang L, Zhao S (2018) Impact of DFIG-based wind farm integration on sub-synchronous torsional interaction between HVDC and thermal generators. IET Gener Transm Distrib 12(17):3913-3923

2. Civelek Z, Lüy M, Çam E, Barışçı N (2016) Control of pitch angle of wind turbine by fuzzy PID controller. Intell Autom Soft Comput 22(3):463-471

3. Van TL, Nguyen TH, Lee D-C (2015) Advanced pitch angle control based on fuzzy logic for variable-speed wind turbine systems. IEEE Trans Energy Convers 30(2):578-587
4. Chilali M, Gahinet P, Scherer C (1996) Multi-objective output-feedback control via LMI optimization. IFAC Proc Vol 29(1):1691-1696

5. Sun Y, Wang L, Li G, Lin J (2010) A review on analysis and control of small signal stability of power systems with large scale integration of wind power. In 2010 International Conference on Power System Technology. IEEE pp. 1-6

6. Kshatriya N, Annakkage UD, Michael Hughes F, Gole AM (2009) Optimized partial eigenstructure assignment-based design of a combined PSS and active damping controller for a DFIG. IEEE Trans Power Syst 25(2):866-876

7. Surinkaew T, Ngamroo I (2017) Two-level coordinated controllers for robust inter-area oscillation damping considering impact of local latency. IET Gener Transm Distrib 11(18):4520-4530

8. Fan L, Yin H, Miao Z (2010) On active/reactive power modulation of DFIG-based wind generation for interarea oscillation damping. IEEE Trans Energy Convers 26(2):513-521

9. Younis MR, Iravani R (2013) Wide-area damping control for interarea oscillations: a comprehensive review. In 2013 IEEE Electrical Power \& Energy Conference. IEEE, pp. 1-6

10. Noureldeen O, Youssef MMM, Hassanin B (2019) Stability improvement of $200 \mathrm{MW}$ Gabal El-Zayt wind farm connected to electrical grid using supercapacitor and static synchronous compensator during extreme gust. SN Appl Sci 1(4):331

11. Ramirez M, Calderon G, Castellanos R (2016) Effect of PODCs for DFIG based wind farms in the inter-area and torsional oscillation damping. IEEE Latin Am Trans 14(8):3648-3654

12. Zhang $X$ et al (2017) Effects of two-area variable inertia on transient stabilisation in interconnected power system with DFIGbased wind turbines. IET Renew Power Gener 11(5):696-706

13. Pokharel $B$ (2011) Modeling, control and analysis of a doubly fed induction generator based wind turbine system with voltage regulation. The Faculty of the Graduate School, Tennessee Technological University (Master thesis). Available at www.seman ticscholar.org. Corpus ID: 107825780, pp 1-336

14. Green M, Limebeer DJ (2012) Linear robust control. Dover Publications, Inc. Mineola, New York

15. Pal BC, Coonick AH, Cory BJ (2001) Linear matrix inequality versus Root-locus approach for damping inter-area oscillations in power systems. Int J Electr Power Energy Syst 23(6):481-489

16. Mohanty A, Viswavandya M, Ray PK, Mohanty S, Mohanty PP (2019) Linear matrix inequality approach in stability improvement through reactive power control in hybrid distributed generation system. IET Smart Grid 2(3):355-363

17. Soliman HM, El Metwally KA (2017) Robust pole placement for power systems using two-dimensional membership fuzzy constrained controllers. IET Gener Trans Distrib 11(16):3966-3973

18. Wu X, Dörfler F, Jovanović MR (2015) Input-output analysis and decentralized optimal control of inter-area oscillations in power systems. IEEE Trans Power Syst 31(3):2434-2444

19. Machowski J, Lubosny Z, Bialek JW, Bumby JR (2020) Power system dynamics: stability and control. Wiley, Hoboken

20. Jafarian M, Ranjbar AM (2013) Interaction of the dynamics of doubly fed wind generators with power system electromechanical oscillations. IET Renew Power Gener 7(2):89-97

21. Mahmud MA, Hossain MJ, Pota HR, Oo AMT (2016) Robust partial feedback linearizing excitation controller design for multimachine power systems. IEEE Trans Power Syst 32(1):3-16

22. Liao K et al (2017) Hybrid fast damping control strategy for doubly fed induction generators against power system inter-area oscillations. IET Renew Power Gener 12(4):463-471

23. Yang $Q$ et al. (2019) Damping inter-area oscillation by fast power modulation of DFIG. 2019 IEEE 8th international conference on advanced power system automation and protection (APAP). IEEE 
24. Cai G et al (2019) A Coordinated dual-channel wide area damping control strategy for a doubly-fed induction generator used for suppressing inter-area oscillation. Appl Sci 9(11):2353

25. Tummala ASLV (2020) A robust composite wide area control of a DFIG wind energy system for damping inter-area oscillations. Prot Control Mod Power Syst 5(1):1-10
Publisher's Note Springer Nature remains neutral with regard to jurisdictional claims in published maps and institutional affiliations. 